\title{
Severe spinal and chest deformity secondary to neurofibromatosis
}

\author{
Lisa Francis, DO • Rajeev Subramanyam, MD (D) Mohamed Mahmoud, MD
}

Received: 5 November 2015/Revised: 10 November 2015/Accepted: 13 November 2015/Published online: 25 November 2015 (C) Canadian Anesthesiologists' Society 2015

A seven-year-old female presented with a history of neurofibromatosis type 1 associated with severe cervicothoracic scoliosis and severe restrictive lung disease. A computerized tomography (CT) scan showed large neurofibromas in the posterior mediastinum and left anterior chest wall, with myelomalacia at the cervicothoracic junction and significant compromise of the right hemithorax (Figure, panel 1). The CT scan also showed that the left third through seventh ribs were markedly remodelled as they coursed towards the dysplastic vertebral bodies (Figure, panel 2). The child was unable to perform a preoperative pulmonary function test. Flexible bronchoscopy performed at a separate pre-scoliosis surgery time period described moderate compression of the trachea just above the carina and severe compression of the right bronchus. The patient subsequently underwent posterior cervical decompression and posterior spinal fusion (C3-T12) due to symptomatic bilateral pain and weakness in the upper and lower extremities. She underwent tracheal extubation without complication $<24 \mathrm{hr}$ after surgery. Postoperatively, her exercise tolerance improved significantly. Panel 3 of the Figure shows an intact spinal instrumentation with the patient standing. Her lower extremity weakness improved substantially, and she currently ambulates without significant pain or an assistive device.

Extensive neuromuscular scoliosis can present many challenges. ${ }^{1}$ Patients undergoing scoliosis surgery require special consideration for airway management. ${ }^{2}$ Intubation in the presence of cervical spine involvement and/or halo traction can be difficult and may increase the risk of neurologic injury. In addition, excessive bleeding and major fluid shifts often occur during surgery. The anesthesiologist must have in-depth knowledge of the critical portions of this procedure (i.e., pedicle screw placement and rod distraction), and hemodynamic stability must be maintained to ensure adequate perfusion to the potentially compromised spinal cord. Hypotension can increase the risk of spinal cord ischemia, which may lead to neurologic deficits, including permanent loss of vision, quadriparesis, or quadriplegia. ${ }^{3}$ A thorough airway assessment, including proper extubation criteria-i.e., cuff leak test, spontaneous respirations, adequate tidal volumes, and purposeful movements, should be obtained prior to attempting extubation.

L. Francis, DO · R. Subramanyam, MD ( $ه)$

M. Mahmoud, MD

Department of Anesthesia, Cincinnati Children's Hospital

Medical Center, University of Cincinnati School of Medicine,

Cincinnati, OH, USA

e-mail: Rajeev.Subramanyam@cchmc.org 

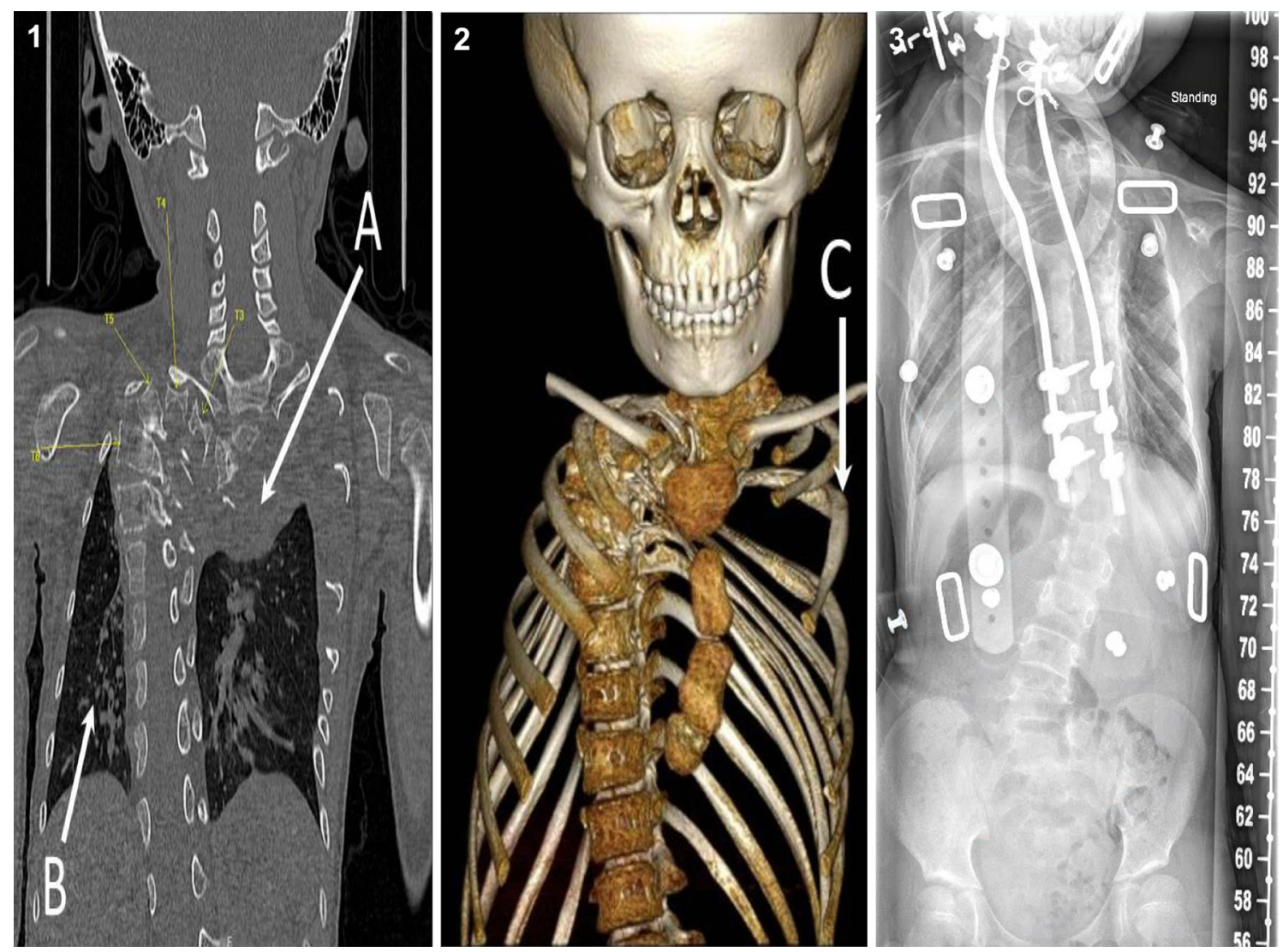

Figure 1) A chest computerized tomography (CT) scan showed large neurofibromas in the posterior mediastinum and left anterior chest wall (arrow A), with myelomalacia at the cervicothoracic junction and significant compromise of the right hemithorax (arrow B). 2) The CT scan showed that the left third through seventh ribs were markedly remodelled as they coursed towards the dysplastic vertebral bodies

\section{Conflicts of interest None declared.}

\section{References}

1. Gibson PR. Anesthesia for correction of scoliosis in children. Anaesth Intensive Care 2004; 32: 548-59. (arrow C). 3) An anterior-posterior $x$-ray of the spine performed approximately $16 \mathrm{mth}$ after surgery with the patient in a standing position reveals a brace, the intact spinal instrumentation, and some residual spinal curvature. There are multiple rib and vertebral anomalies observed on the upper chest and thoracic spine

2. Calder I, Calder J, Crockard HA. Difficult direct laryngoscopy in patients with cervical spine disease. Anaesthesia 1995; 50: 75663.

3. DiCindio S, Schwartz DM. Anesthetic management for pediatric spine fusion: implications of advances in spinal cord monitoring. Anesthesiol Clin North America 2005; 23: 765-87. 